\title{
Exploring the Energy-Time Tradeoff in MPI Programs on a Power-Scalable Cluster
}

\author{
Vincent W. Freeh \\ Feng Pan Nandani Kappiah \\ Department of Computer Science \\ North Carolina State University \\ \{vwfreeh,fpan2,nkappia\}@ncsu.edu
}

\author{
David K. Lowenthal \\ Rob Springer \\ Department of Computer Science \\ The University of Georgia \\ \{dkl,springer\}@cs.uga.edu
}

\begin{abstract}
Recently, energy has become an important issue in highperformance computing. For example, supercomputers that have energy in mind, such as BlueGene/L, have been built; the idea is to improve the energy efficiency of nodes. Our approach, which uses off-the-shelf, high-performance cluster nodes that are frequency scalable, allows energy saving by scaling down the CPU.

This paper investigates the energy consumption and execution time of applications from a standard benchmark suite (NAS) on a power-scalable cluster. We study via direct measurement and simulation both intra-node and inter-node effects of memory and communication bottlenecks, respectively. Additionally, we compare energy consumption and execution time across different numbers of nodes.

Our results show that a power-scalable cluster has the potential to save energy by scaling the processor down to lower energy levels. Furthermore, we found that for some programs, it is possible to both consume less energy and execute in less time when using a larger number of nodes, each at reduced energy. Additionally, we developed and validated a model that enables us to predict the energy-time tradeoff of larger clusters.
\end{abstract}

\section{Introduction}

Recently, power-aware computing has gained traction in the high-performance computing (HPC) community. As a result, low-power, high-performance clusters, such as BlueGene/L [1] or Green Destiny [32], have been developed to stem the ever-increasing demand for energy. Such systems improve the energy efficiency of nodes. Consider the case of Green Destiny - a cluster of Transmeta processors-which consumes less energy than a conventional supercomputer. In particular, Green Destiny consumes about three times less energy per unit performance than the ASCI Q machine.
However, because Green Destiny uses a slower (and cooler) microprocessor, ACSI Q is about 15 times faster per node (200 times overall) [32]. A reduction in performance by such a factor surely is unreasonable from the point of view of many users. If performance is the only goal, then one should continue on the current "performance-at-all-costs" path of HPC architectures. On the other hand, if power is paramount, then one should use a low-performance architecture that executes more instructions per unit energy.

We believe one should strike a path between these two extremes. This is conceptually possible because an increase in CPU frequency generally results in a smaller increase in application performance. The reason for this is that the CPU is not always the bottleneck resource. Therefore, increasing frequency also increases CPU stalls-usually waiting for memory or communication. Consequently, there are opportunities where energy can be saved, without an undue performance penalty, by reducing CPU frequency.

As a preliminary step, this paper studies the tradeoff between power and performance (or equivalently energy and time) for HPC. In particular, this paper makes two key contributions. First, we evaluate a real, small-scale powerscalable cluster, which is a cluster composed of processors that are each frequency scalable-i.e., their clock speed and hence power consumption can be changed dynamically. This illustrates the tradeoff between the power-conserving benefits and the time-increasing costs of such a machine. Second, in order to estimate the potential for large powerscalable clusters, we developed a simulation model that allows us to predict energy consumed and time taken. Studies like this are needed so that architects can make informed decisions before building or purchasing large, expensive power-scalable clusters.

We used the NAS benchmark suite for evaluation. Our results show that the potential for energy savings from using a slower gear depends on the benchmark. In particular, we found that on one node, it is possible to use $10 \%$ less energy while increasing time by $1 \%$, with CG. However, with EP there was essentially no savings. We present 
a simple metric that predicts this energy-time tradeoff. Additionally, we found that in some cases one can save energy and time by executing a program on more nodes at a slower gear rather than on fewer nodes at the fastest gear. We believe this will be important in the future, where a program running on a cluster may be allowed to generate only a fixed amount of heat. Finally, this paper presents a model for estimating energy consumption. It validates the model on a real power-scalable cluster, then extrapolates to determine the benefit of a larger power-scalable cluster.

The rest of this paper is organized as follows. Section 2 describes related work. Next, Section 3 discusses the measured results on our power-scalable cluster. The next section presents our simulation results up to 32 nodes. Finally, Section 5 summarizes and describes future work.

\section{Related Work}

There has been a voluminous amount of research performed in the general area of energy management. In this section we describe some of the closely related research. We divide the related work into two categories: server/desktop systems and mobile systems.

\subsection{Server/Desktop Systems}

Several researchers have investigated saving energy in server-class systems. The basic idea is that if there is a large enough cluster of such machines, such as in hosting centers, energy management can become an issue. In [5], Chase et al. illustrate a method to determine the aggregate system load and then determine the minimal set of servers that can handle that load. All other servers are transitioned to a low-energy state. A similar idea leverages work in cluster load balancing to determine when to turn machines on or off to handle a given load [26, 27]. Elnozahy et al. [10] investigated the policy in [26] as well as several others in a server farm. Such work shows that power and energy management are critical for commercial workloads, especially web servers [3, 21]. Additional approaches have been taken to include DVS [9, 28] and request batching [9]. The work in [28] applies real-time techniques to web servers in order to conserve energy while maintaining quality of service.

Our work differs from most prior research because it focuses on HPC applications and installations, rather than commercial ones. A commercial installation tries to reduce cost while servicing client requests. On the other hand, an HPC installation exists to speedup an application, which is often highly regular and predictable. One HPC effort that addresses the memory bottleneck is given in [18]; however, this is a purely static approach.

In server farms, disk energy consumption is also significant. One study of four energy conservation schemes concludes that reducing the spindle speed of disks is the only viable option for server farms [4]. DRPM is a scheme that dynamically modulates the speed of the disk to save energy [14, 15]. Another approach is to improve cache performance-if many consecutive disk accesses are cache hits, the disk can be profitably powered down until there is a miss; this is the approach taken by [36]. An alternative is to use an approach based on inspection of the program counter [12]; the basic idea is to infer the access pattern based on inspection of the program counter and shut down the disk accordingly. A final approach is to try to aggregate disk accesses in time. A compiler/run-time approach using this was designed and implemented in [16], and a prefetching approach in [25]. Both were designed for mobile systems but can be directly applied to server/desktop systems.

There are also a few high-performance computing clusters designed with energy in mind. One is BlueGene/L [1], which uses a "system on a chip" to reduce energy. Another is Green Destiny [32], which uses low-power Transmeta nodes. A related approach is the Orion Multisystem machines [23], though these are targeted at desktop users. However, using a low-power processor sacrifices performance in order to save energy.

\subsection{Mobile Systems}

There is also a large body of work in saving energy in mobile systems; most of the early research in energy-aware computing was on these systems. Here we detail some of these projects.

At the system level, there is work in trying to make the OS energy-aware through making energy a first class resource $[29,8,6]$. Our approach differs in that we are concerned with saving energy in a single program, not a set of processes. One important avenue of application-level research on mobile devices focuses on collaboration with the OS (e.g., $[22,33,35,2])$. Such application-related approaches are complementary to our approach.

In terms of research on device-specific energy savings, there is work in the CPU via DVS (e.g., $[11,13,24])$, the disk via spindown (e.g., $[17,7])$, and on the memory or network [20,19]. The primary distinction between these projects and ours is that energy saving is typically the primary concern in mobile devices. In HPC applications, performance is still the primary concern.

\section{Measured Results}

This section describes the results of our experiments using our power-aware cluster. We studied the programs in the NAS parallel benchmark suite on all valid configurations using up to 9 nodes. Presumably, such mature benchmarks have been thoroughly analyzed and are well-written (e.g., see [34])—so that they are not unrealistically communication bound. 


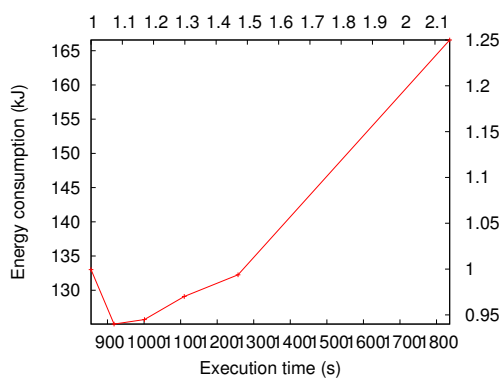

(a) BT B

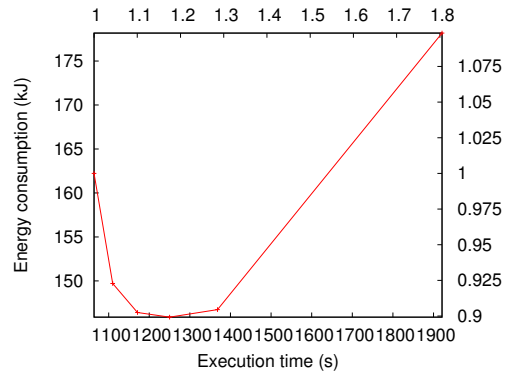

(d) LU B

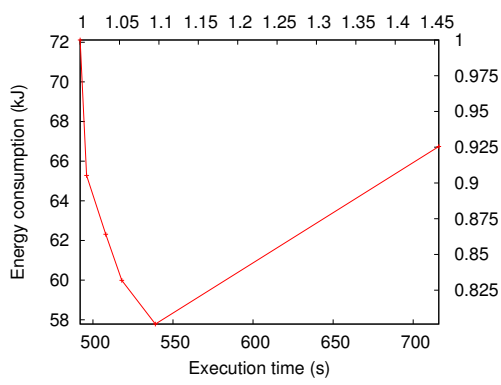

(b) CG B

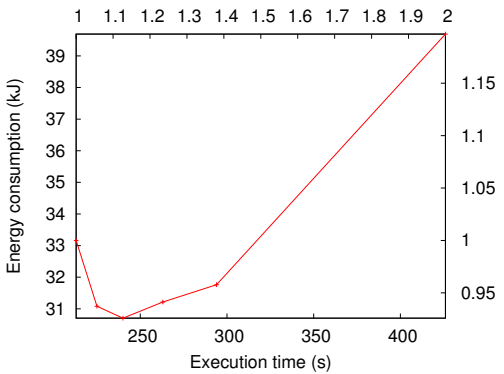

(e) MG B

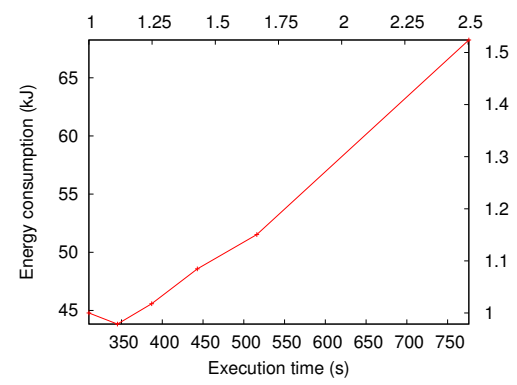

(c) EP B

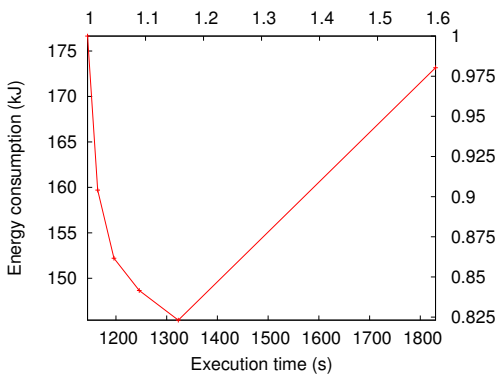

(f) SP B

Figure 1. Energy consumption vs execution time for NAS benchmarks on a single AMD machine.

This study uses, as a reference example, a cluster of ten nodes, each equipped with a frequency- and voltagescalable AMD Athlon-64. We run each program at each available energy gear: $2000 \mathrm{MHz}, 1800 \mathrm{MHz}, 1600 \mathrm{MHz}$, $1400 \mathrm{MHz}, 1200 \mathrm{MHz}$, and $800 \mathrm{MHz} .{ }^{1}$ The voltage, which ranges from $1.5-1.0 \mathrm{~V}$, is reduced in each gear. Each node has 1GB main memory, a $128 \mathrm{~KB} \mathrm{L1}$ cache (split), and a $512 \mathrm{~KB} \mathrm{~L} 2$ cache, and the nodes are connected by $100 \mathrm{Mb} / \mathrm{s}$ network. In this paper, we control the CPU power and measure overall system energy. This is effective in saving energy because the CPU-a major power consumer-uses less power. In particular, the Athlon CPU used in this study consumes approximately $45-55 \%$ of overall system energy. ${ }^{2}$

For each program we measure execution time and energy consumed for an application at a range of energy gears. Execution time is elapsed wall clock time. The voltage and current consumed by the entire system is measured by precision multimeters at the wall outlet to determine the instantaneous power (in Watts). This value is integrated over time to determine the energy used. Integration is performed

1 The $1000 \mathrm{MHz}$ does not work reliability on a few of the nodes.

2 CPU power is not measured directly. However, the system power at the fastest energy gear is 140-150 W. The AMD datasheet states that the maximum CPU power consumption is $89 \mathrm{~W}$. We estimate the peak power of the CPU for our application is in the range of 70-80 W, which is $45-55 \%$ of system power. by a separate computer that samples two multimeters several tens of times a second.

We divide our results into two parts. First, we discuss results on a single processor. This shows the energy-time tradeoff due to the memory bottleneck. The next section shows time and energy results for the NAS suite on multiple nodes. This shows the effect of the communication bottleneck as well as the energy-time tradeoff when the number of nodes increases.

\subsection{Single Processor Results}

Figure 1 shows the results of executing 6 NAS programs on a single Athlon-64 processor. The NAS FT benchmark is not shown because we cannot get it to work, and IS is not shown because (1) class B is too small to get any parallel speedup and (2) class $C$ thrashes on 1 and 2 nodes, making comparative energy results meaningless. For each graph, the total system energy consumed at each gear (gear 1 is fastest, gear 6 is slowest) is plotted on the y-axis and the total execution time is plotted on the $\mathrm{x}$-axis. The higher of two points uses more energy, and the further right of two points takes more time. Therefore, a near-vertical slope indicates an energy savings with little time delay between adjacent gears, whereas a horizontal slope indicates a time penalty and no energy savings. For readability, the origin of the graphs is 
not $(0,0)$. Therefore, the alternate axes show the time and energy relative to the fastest gear (leftmost point).

All of our tests show that for a given program, using the fastest gear takes the least time (i.e., it is the leftmost point on the graph). The greatest relative savings in energy is $20 \%$, which occurs in CG operating at gear $5(1200 \mathrm{MHz})$. This savings incurs a delay (increase in execution time) of almost $10 \%$. The best savings relative to performance occurs at gear $2(1800 \mathrm{MHz})$ which saves $9.5 \%$ energy with a delay less than $1 \%$. On the other hand, EP at $1800 \mathrm{MHz}$ saves $2 \%$ energy with an $11 \%$ delay. This delay is approximately the same as the increase is CPU clock cycle.

At a lower gear, a given program runs longer; if the decrease in power exceeds the increase in time, a lower gear uses less energy. We have found that the increase in time from one gear to another is bounded above and below as follows: $1 \leq \frac{T_{i+1}}{T_{i}} \leq \frac{f_{i}}{f_{i+1}}$, where $T_{i}$ and $f_{i}$ are the execution time and frequency at gear $i$, respectively. In words, shifting to a slower gear will never speed up a program, nor will it slow a program by more than the increase in CPU cycle time. This makes intuitive sense and is borne out by our empirical results. Further testing shows that a program with a time increase close to the upper bound has the CPU on the critical path. In other words, its performance depends on the throughput of the CPU. EP is representative of such a program. On the other hand, a program such as CG, which has a small time increase at slower gears, is largely independent of CPU frequency. Because we tested the in-core version of NAS (i.e., class B), these programs do not have significant I/O. Therefore, programs that are not dependent on the CPU do not have the CPU on the critical path, e.g., the memory subsystem is instead on the critical path. One more test that also shows the dependency on CPU or memory is the effect the gear has on overall UPC (micro-operations per cycle). In memory-bound applications, the UPC increases as frequency decreases. This increase is because the cycle time of the processor increases, but the memory latency remains the same. At a lower frequency, memory latency is less in terms of CPU cycles. Consequently, there fewer delay slots that must be filled, and ILP increases.

We looked for a predictor of the energy-time tradeoff. The typical measure of IPC or UPC is problematic because it varies too much. The programs we consider in this section do not perform much I/O. Therefore, the memory system is the only other component that could be on the critical path. The metric we chose is micro-operations per memory reference (i.e., per L2 cache miss). This value stays constant as the frequency changes. What UPM ( $\mu \mathrm{op} / \mathrm{miss})$ indicates is the pressure exerted on the memory system by the program. Misses per cycle or per second are dependent on CPU frequency and, therefore, are not useful.

Table 1 shows the ratio UPM for the 6 NAS benchmark programs. The benchmarks are sorted from highest to lowest, ranging from a high of 844 for EP to a low of 8.60 for

\begin{tabular}{|c||c|c|c|}
\hline & UPM & Slope $1 \rightarrow 2$ & Slope $2 \rightarrow 3$ \\
\hline EP & 844. & -0.189 & 0.288 \\
BT & 79.6 & -0.811 & 0.0510 \\
LU & 73.5 & -1.78 & -0.355 \\
MG & 70.6 & -1.11 & -0.161 \\
SP & 49.5 & -5.49 & -1.52 \\
CG & 8.60 & -11.7 & -1.69 \\
\hline
\end{tabular}

Table 1. Predicting energy-time tradeoff.

CG. The next column shows the slope of the energy-time curve from the fastest gear to gear 2, computed as $\frac{E_{2}-E_{1}}{T_{2}-T_{1}}$. A large negative number indicates a near vertical slope and a significant energy savings relative to the time delay. On the other hand, a small negative number indicates a near horizontal slope and little energy savings. The last column shows the slope from second to third gear. Except for MG, we see that the slopes are also sorted, in this case from greatest (positive) to least (negative). Because the more negative slope indicates a better energy-time tradeoff, this table shows that memory pressure tends to predict the energytime tradeoff.

\subsection{Multiple Processor Results}

The previous section investigated the energy-time tradeoff on a single node. This section studies the effect of distributed programs. Figure 2 shows results from six NAS programs. Results from FT and IS are not shown for the reasons stated above. Each graph has the same general layout as in Figure 1, except that it shows the results from multiple experiments: 2, 4, and 8 nodes (or 4 and 9 nodes in the case of BT and SP). One-node results from the previous section are also plotted, but in most cases these curves are to the right window shown. The energy plotted is cumulative energy of all nodes used.

Before discussing the results, we describe the possible shapes of these graphs. First, for a fixed number of nodes, the shape of the curve depends on the memory and communication bottlenecks. This is because in a distributed program, not only might a processor wait for the memory subsystem, but at times it also might block awaiting a message. In either scenario, the CPU is not on the critical path, and idle or slack time is more efficiently spent at a lower energy gear.

Second, consider the possible effects when comparing an experiment with $2 P$ nodes versus one with $P$ nodes. The following possibilities exist. Note that we do not consider the case where the time on $2 P$ nodes is larger than on $P$ nodes.

1. The curve for $2 P$ nodes can lie completely above and to the left of the curve for $P$ nodes. Each point on the $2 P$ node curve lies above all points on the $P$ node 


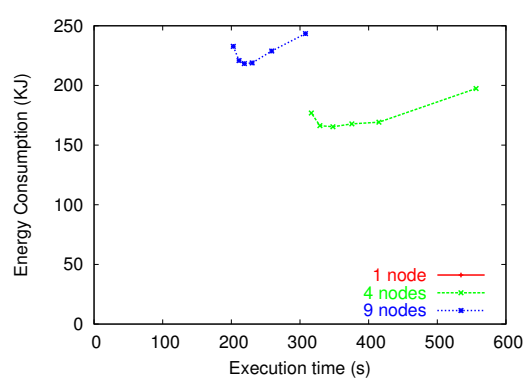

(a) BT B

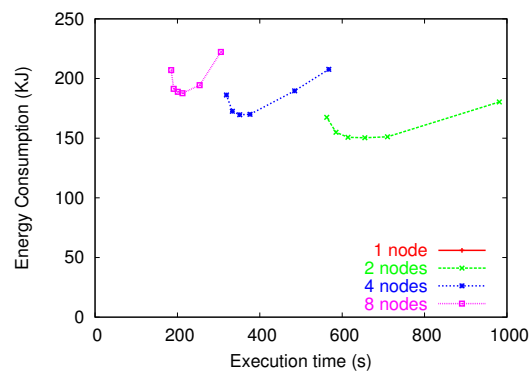

(d) LU B

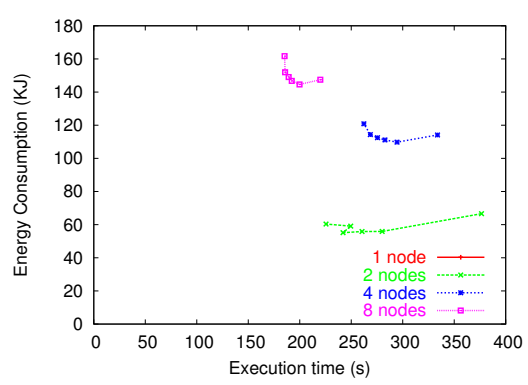

(b) CG B

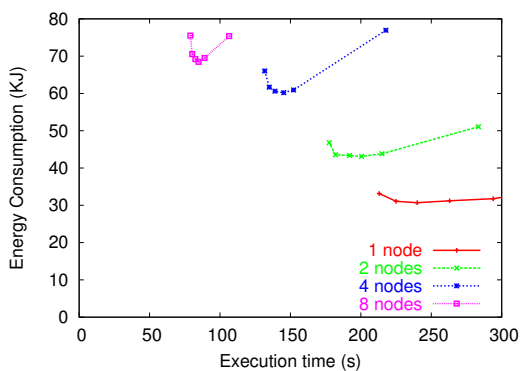

(e) MG B

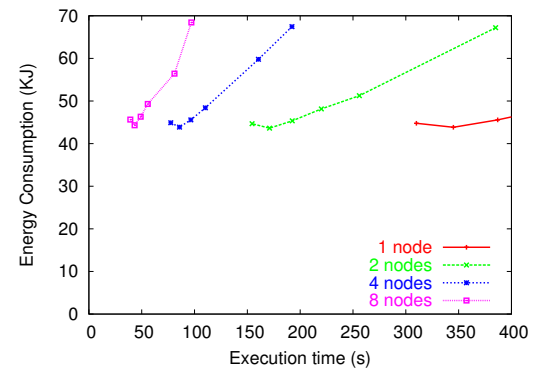

(c) EP B

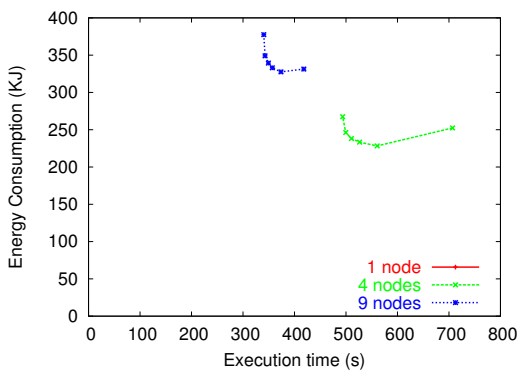

(f) SP B

Figure 2. Energy consumption vs execution time for NAS benchmarks on 2, 4, and 8 (or 2 and 9) nodes.

curve. This case occurs when the program achieves poor speedup on $2 P$ nodes compared to $P$ nodes.

2. The point that represents the fastest energy gear for $2 P$ nodes can be to the left, at or below, the corresponding point on the curve for $P$ nodes. This case occurs when the program achieves perfect or superlinear speedup on $2 P$ nodes compared to $P$ nodes.

3. The curve for $2 P$ nodes can lie to the left of the curve for $P$ nodes, but not completely above or below the fastest gear point for $P$. This is the most interesting case. While the program executes faster and consumes more energy in the fastest gear on $2 P$ nodes than on $P$ nodes, there is a lower gear at $2 P$ nodes that has less energy consumption than the fastest gear point at $P$ nodes. Therefore, it is possible to achieve better execution time and lower energy consumption by running at a lower energy gear on $2 P$ nodes than at a higher energy gear on $P$ nodes. There is not an energy-time tradeoff between these points because one point dominates the other in both energy and time. This case occurs case when speedup is good (i.e., not superlinear and not poor) and there are a significant number of main memory accesses (so that scaling down the pro- cessor has only a slightly detrimental effect).

We describe each of the cases in turn below.

\section{Case 1: Poor Speedup}

Figure 2 offers several examples of case 1. In particular, this case is illustrated in BT, SP, and MG from 2 to 4 nodes, and CG from 4 to 8 nodes.

We believe in the future a given supercomputer cluster will be restricted to a certain amount of power consumption or heat dissipation. If there is a limit for energy/power consumption or heat dissipation, this would be represented as a horizontal line. For programs in this case, the line will intersect at most one of the curves. The most desirable point would be the leftmost (fastest) one under the limit.

\section{Case 2: Superlinear Speedup}

Figure 2 does not contain an example of superlinear speedup. However, EP, which gets almost perfect speedup, illustrates this case. Power consumption doubles when the number of nodes doubles. Because the time is cut in half, the total energy consumed is the same. With superlinear 


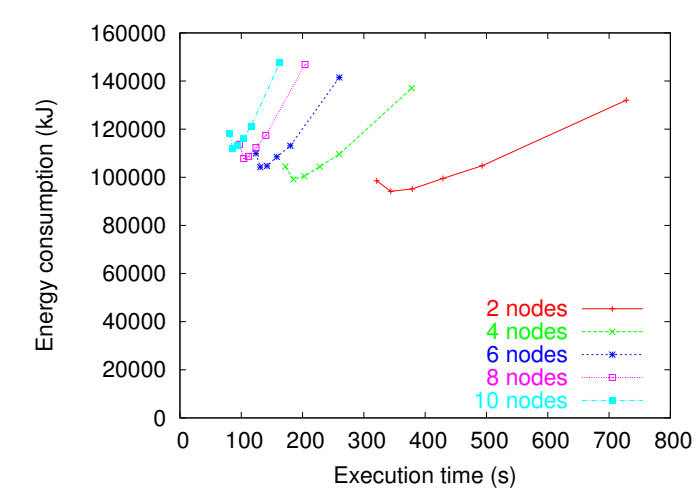

Figure 3. Energy consumption vs. execution time for Jacobi iteration on 2, 4, 6, 8, and 10 nodes.

speedup the energy consumption decreases as nodes are added. When speedup is perfect or superlinear there is no energy-time tradeoff, because the energy and time are never better with fewer nodes.

\section{Case 3: Good Speedup}

Figure 2 shows several examples of this case. First, consider LU at 4 and 8 nodes. Gear 4 on 8 nodes uses approximately the same energy as the fastest gear on 4 nodes, but executes $50 \%$ more quickly. The fastest gear on 8 nodes executes $72 \%$ faster than on 4 nodes, but uses $12 \%$ more energy. This case illustrates an additional choice not available in a conventional cluster, which only supports either the fastest gear option ( 4 or 8 nodes). So a user must trade off a performance increase against an energy increase. With a power-scalable cluster, the user can select a slower gear on 8 nodes, which may offer better performance for the same energy consumption. Thus, a user of a power-scalable cluster has two dimensions to explore: (1) number of nodes and (2) processor performance gear. In case 3 , the user may be able to get better performance by using more nodes, with each node executing at a lower energy gear.

Next, Figure 3 plots data for a (hand-written) Jacobi iteration application. This application is shown because it can run at any number of nodes, unlike the NAS benchmarks. The figure shows energy-time curves on 5 configurations: $2,4,6,8$, and 10 nodes. Because this application gets good speedup $(1.9,3.6,5.0,6.4$, and 7.7) each adjacent pair of curves falls in case 3 . For example, executing in second or third gear on 6 nodes results in the program finishing slightly faster and using less energy than using first gear on 4 nodes.

Finally, we present results from a synthetic benchmark. This benchmark models CG in terms of its cache miss rate, but achieves good speedup (over 7 on 8 nodes). The pur-

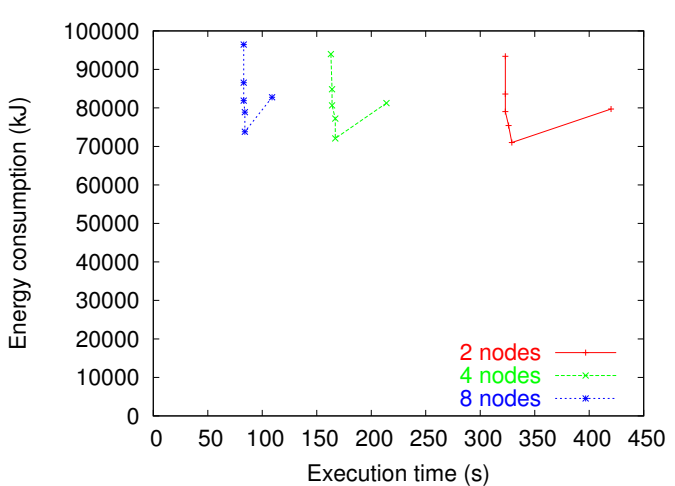

Figure 4. Synthetic benchmark with high memory pressure.

pose of this benchmark is to show the potential of a powerscalable cluster. Figure 4 shows the results. Because the miss rate is high (7\%), the execution time penalty for scaling down is low (e.g., 3\% at gear 5, 1200MHz), and the corresponding energy savings is large (e.g., 24\% at gear 5). Furthermore, compared to gear 1 on 4 nodes, gear 5 on 8 nodes uses $80 \%$ of the energy and executes in half the time.

\section{Simulated Results}

The previous section presented results on up to ten power-scalable nodes. While the results are encouraging, it is left unclear what performance (in time and energy) can be expected for larger power-scalable clusters. Most likely, before building or buying a large power-scalable cluster, one would like to determine the performance potential. As we do not have access to more than ten nodes, this section seeks to address this issue by developing a simulation model.

\subsection{Model}

Understanding scalability of parallel programs is of course a difficult problem; indeed, it is one of the fundamental problems in parallel computing [30, 31]. Researchers try to predict scalability using a range of techniques, from analytical to execution-based. We will use a combination of both.

Our methodology for predicting the behavior of larger power-aware clusters (that we cannot run programs on) is as follows. We model computation and communication using a combination of Amdahl's law, trace gathering, and source code inspection. To assist in understanding scalability, we use results from a 32-node (non-power-scalable) cluster. After that, we use the model to predict execution time on up to 32 power-scalable nodes at the fastest gear. (Our methodology can be applied to larger instances, but we do not have 
reliable results from a larger non-power-scalable cluster.) To determine time and energy on slower gears, we measure power consumption on a cluster node and use a straightforward algebraic formula. Below we describe our five-step methodology in full detail.

Step 1: Gather time traces. The first step is to gather active and idle times on $n$ nodes $\left(T^{A}(n)\right.$ and $T^{I}(n)$, respectively, where $T^{I}(n)$ includes the actual communication time) on each of our clusters. This includes the (ten-node) powerscalable cluster described above as well as a 32-node Sun cluster. $^{3}$ The parameter $n$ varies to include all configurations on which the NAS suite can run. We gather the traces at only the fastest gear on the power-scalable cluster, and the Sun cluster is not power scalable. We decompose the total execution time into $T^{A}(n)$ and $T^{I}(n)$ by instrumenting MPI. This instrumentation intercepts all relevant MPI calls, and writes a timestamp to a log file.

For all MPI communication routines used in each benchmark, interception functions report the time at which the routine was entered and exited. These operations create a trace from which we recover active and idle times. To reduce perturbation, each trace record is written to a local buffer.

Step 2: Model computation and communication. The second step is to develop a model of computation and communication that is based on $T^{A}(n)$ and $T^{I}(n)$. This will help us predict (in step 3) $T^{A}(m)$ and $T^{I}(m)$ where $m>10$, i.e, for power-scalable configurations with more than ten nodes. Our approach here is distinct for each quantity, out of necessity: no matter what the gear, the power consumed is different when computing than when blocking awaiting data. The formulas below do not mention gear because all of these measurements are taken at the fastest gear.

Determining $F_{p}$ and $F_{s}$. Here, we use Amdahl's law to estimate $F_{p}$ and $F_{s}$, which denote the parallelizable and inherently sequential fractions of an application, respectively. For a test with $i$ nodes, we estimate $F_{p}$ and $F_{s}$ as follows:

$$
\begin{aligned}
T^{A}(i) & =T^{A}(1)\left(F_{p} / i+F_{s}\right) \\
F_{p} & =1-F_{s}
\end{aligned}
$$

We obtain a family of $F_{p}$ and $F_{s}$ values. We will use these to determine $F_{p}$ and $F_{s}$ on large power-scalable clusters. Also, $T^{A}(n)$ represents the maximum computation time over all nodes.

Classifying communication. Here, we recall that $T^{I}$ includes idle time and communication time. While idle time (due to load imbalance) can be directly derived from $T^{A}$, the communication cost cannot. Hence, our approach is to categorize communication of each NAS program into one of three groups: logarithmic, linear, or quadratic. These are

3 We also ran tests on a 64-node Xeon cluster, but as the network was shared among several large jobs, the results were unreliable. three common scaling behaviors for communication. To do this, we rely on three complementary methods: (1) inspection of the behavior of our measured $T^{I}$ on up to nine power-scalable nodes, (2) dynamic measurement of number of each MPI call as well as inspection of corresponding source code, and (3) the literature in the field (e.g., [34]). Specifically, we classified communication in BT, EP, MG, and SP as logarithmic; CG as quadratic, and LU as linear.

Step 3: Extrapolation of $T^{A}(m)$ and $T^{I}(m)$ at fastest gear. Third, we extrapolate to 16,25 , and 32 power-scalable nodes, i.e., $m>10$. For a given number of nodes, $m$, the sum of $T^{A}(m)$ and $T^{I}(m)$ yields the execution time.

Predicting active time: Predicting $T^{A}(m)$, requires an appropriate $F_{p}$ and $F_{s}$ for 16 and 32 nodes on the powerscalable cluster. Using our measured values on up to 32 nodes on the Sun cluster and up to 9 nodes on our powerscalable cluster, we fit $F_{p}$ and $F_{s}$ for 16,25 , and 32 nodes on the power-scalable cluster using a linear regression.

Predicting idle time: Given the classification of communication behavior (logarithmic, linear, or quadratic) for each application, we use regression to fit a curve to the communication using measured data on power-scalable nodes. This gives us communication time on 16, 25, and 32 nodes.

Validation. Our technique is validated in the following way. For $T^{A}(m)$, we compared $F_{p}$ and $F_{s}$ on up to 9 nodes on both clusters. With only 1 exception, it was identical; the outlier was $\mathrm{CG}$, where the parallelism actually increases from 4 to 8 nodes on our power-scalable cluster, but is constant on the Sun cluster. For $T^{I}(m)$, each communication shape that we chose for our power-scalable cluster is identical on the Sun cluster up to 32 nodes. We also note that [34] supports our conclusion on five of the six programs. The exception is LU; for this program, we found that communication was best modeled as a constant; our traces showed that when nodes are added, each node sends more messages, but the average message size decreases.

Armed with estimates of $T^{A}(m)$ and $T^{I}(m)$ on larger configurations (at the fastest gear), we now turn our attention to determining the effect of different energy gears on execution time and energy consumption. The last two steps in this methodology are concerned with this issue.

Step 4: Determine $S_{g}, P_{g}$, and $I_{g}$. The next step is to gather power data from a single power-scalable node. Two values will be needed on a per-application and per-gear basis: application slowdown $\left(S_{g}\right)$ and average power consumption $\left(P_{g}\right)$. Separately, the power consumption for an idle (i.e., inactive) system is determined for each gear $\left(I_{g}\right)$.

This data determines the increase in time and the decrease in power. The execution time for a sequential program is wall clock time. This is done for each (sequential) program at each energy gear. The ratio $S_{g}$ is determined as follows: $S_{g}=\frac{T_{g}(1)-T_{1}(1)}{T_{1}(1)}$. Now that we are discussing gear, we modify our notation: $T_{g}(1)$ is the time on one node 
at gear $g$.

The values $P_{g}$ and $I_{g}$ are obtained by measuring overall system power. The voltage and current consumed by the entire system is measured at the wall outlet to determine the instantaneous power (in Watts), as described in Section 3. This experimental setup determines the values, $P_{g}$, for each application and for each gear. The same setup, except this time with no application running, was used to determine the power usage of an idle system $\left(I_{g}\right)$ at each gear.

Step 5: Determine $T_{g}(m)$ and $E_{g}(m)$. The final step is to estimate the time and energy consumption of a powerscalable cluster using the information developed so far. The time for a lower gear is computed by increasing the active time by the appropriate ratio, $S_{g}$. We assume that executing in a reduced gear does not itself increase the idle time, as our experimentation has shown that the time for communication is independent of the energy gear-the computational load during MPI communication is quite low. Using the values of $P_{g}$ and $I_{g}$, we can estimate energy consumption at each lower gear for the MPI program. In this straightforward case, the time and energy estimates, on $m$ nodes, for each gear are:

$$
\begin{aligned}
& T_{g}(m)=S_{g} T^{A}(m)+T^{I}(m) \\
& E_{g}(m)=P_{g} S_{g} T^{A}(m)+I_{g} T^{I}(m) .
\end{aligned}
$$

At slower gears the compute time is greater than that of the fastest gear, whereas the idle time is independent of the gear. Thus the time executing in gear $g$ increases to $S_{g} T^{A}$. Communication latency is independent of gear, so this is assumed to remain the same.

However, this naive case above is too simple because it assumes all computation is on the critical path. In many programs, not all computation is on the critical path. Of course, reducing the energy gear of any computation on the critical path will delay other nodes, who must wait for data sent from the (now slower) node. In the refined model, $T^{A}$ is classified into what critical and reducible work $\left(T^{C}\right.$ and $T^{R}$ ), and in our estimates of computation we separate these and estimate each. In short, executing reducible work in a slower gear might not increase overall execution time, because an increase in the time of reducible work will decrease the idle time. On the other hand, executing critical work in a slower gear always increases execution time. The communication latency, which is unaffected by CPU frequency, is delayed only by the slowdown applied to the critical work. The idle time is slack for the reducible work. However, if reducible work is slowed such that all slack is consumed, then the time will be extended. This point of inflection is when $T^{R}+T^{I}=S_{g} T^{R}$.

The post-processing analysis conservatively determines the reducible work to be computation between the last send ${ }^{4}$

4 We assume that the send is asynchronous. and a blocking point. In between those two points there is no interaction between nodes, so the work is not on the critical communication path. With this refinement, Equation (1) changes as shown below. Note that for notational simplicity, we omit number of nodes $(m)$.

$T_{g}=\left\{\begin{array}{lr}S_{g}\left(T^{C}+T^{R}\right), & \text { if } T^{I}+T^{R} \leq S_{g} T^{R} \\ S_{g}\left(T^{C}+T^{R}\right)+T^{I}+T^{R}-S_{g} T^{R}, \text { otherwise }\end{array}\right.$

Then, Equation (2) becomes

$E_{g}=\left\{\begin{array}{lr}P_{g} S_{g}\left(T^{C}+T^{R}\right), \quad \text { if } T^{I}+T^{R} \leq S_{g} T^{R} \\ P_{g} S_{g}\left(T^{C}+T^{R}\right)+I_{g}\left(T^{I}+T^{R}-S_{g} T^{R}\right), \\ & \text { otherwise }\end{array}\right.$

Assumptions The methodology described in this section makes use of two assumptions. First, this methodology assumes that the power consumed when an application is computing is constant (because we use the average power). This is reasonable as the observed power consumption for regular applications is fairly constant (within a few percent). Second, it assumes that the power consumption follows a step function, in that at all times the power consumed is either $P_{g}$ or $I_{g}$. In reality, the transition takes between these power levels takes some time. However, this time is negligible, and the transition occurs on both sides of an idle period, tending to equal out.

\subsection{Evaluation}

Figure 5 shows the results of our simulation on each application ranging from 2-32 nodes. All node configurations up to and including 9 nodes are actual runs on the cluster, and configurations of 16,25 , and 32 nodes are simulated using the model discussed previously.

In the same way that the eight- and nine-node tests tend to be more "vertical" than the two- and four-node tests, as with the runs up to nine nodes, the shapes of the graphs tend to become more "vertical" when using 16, 25, or 32 nodes; i.e., using lower gears becomes a better idea. As an example, consider SP. On four nodes, second gear consumes the least energy. On the other hand, on 16 nodes, fourth gear consumes the least energy.

One possible implication of this is that for massively parallel power-scalable clusters, the individual nodes can be placed in a relatively low energy gear with only a modest time penalty. As discussed in the previous section, this may potentially allow for supercomputing centers to fit more nodes in a rack while staying within a given power budget. On the other hand, this could degrade performance significantly if many applications for such machines are embarrassingly parallel.

Second, speedup on the NAS suite generally starts to tail off around 25 or 32 nodes. Again, this is because this benchmark suite uses non-scaled speedup. The result of this is that 


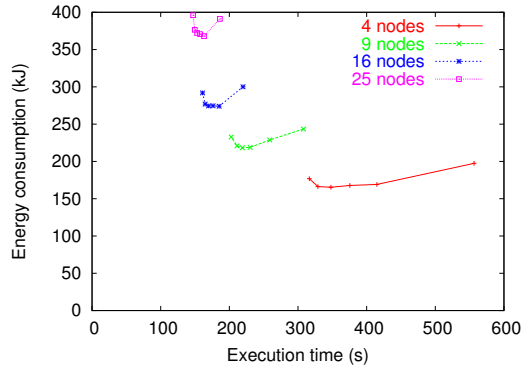

(a) BT B

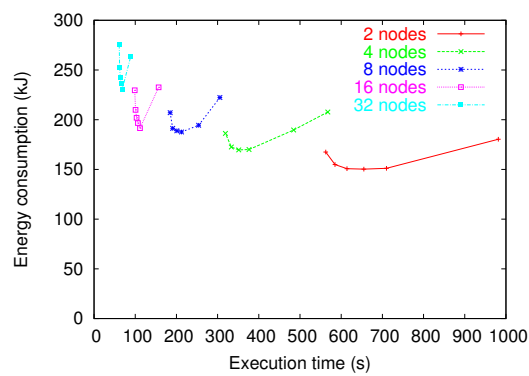

(d) LU B

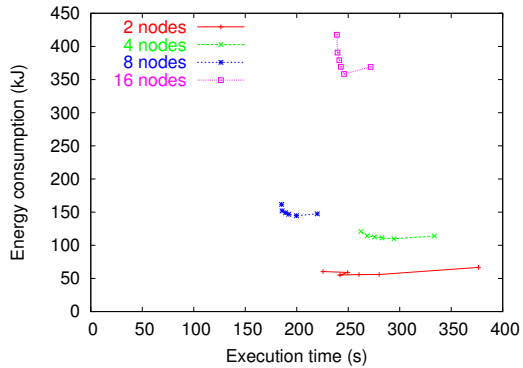

(b) CG B

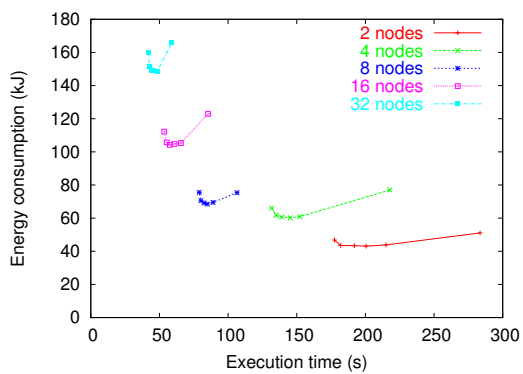

(e) MG B

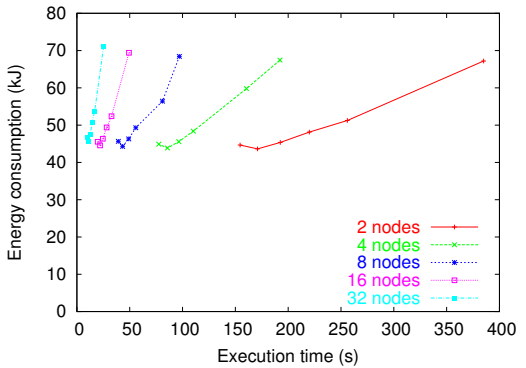

(c) EP B

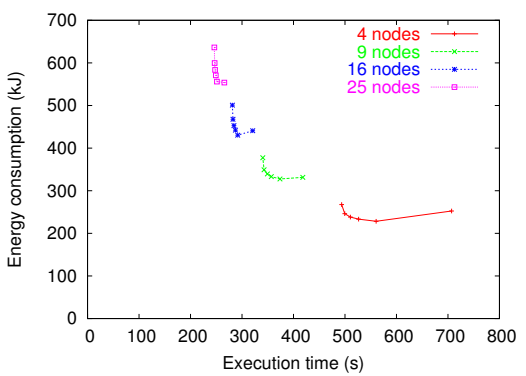

(f) SP B

Figure 5. Simulated energy consumption vs execution time for NAS benchmarks on up to 32 nodes.

the total cluster energy consumed starts to increase dramatically. Essentially, continuously increasing the number of nodes causes an application to be placed in the poor speedup classification (see previous section), which we know is energy inefficient. Also, for each application, there exists a certain number of nodes that, if exceeded, will cause program slowdown. It appears that that point is around 32 nodes for the NAS suite on our power-scalable Athlon-64 cluster.

This problem is not unique to power-aware computing; indeed, it is a problem with roots in the scalability field. However, it is clear that when this phenomenon occurs, it is necessarily the case that communication dominates computation. This means that in fact a lower gear is almost certain to be better. Hence, if one does not know what the parallel efficiency for a given application is, using a lower energy gear is a safeguard against excessive energy consumption.

\section{Conclusions and Future Work}

This paper has investigated the tradeoff between energy and performance in MPI programs. We have studied trends on both one processor and multiple processor programs. Using the NAS benchmark suite, we found for example that on one node, it is possible to use $10 \%$ less energy while increasing time by $1 \%$. Additionally, we found that in some cases one can save energy and time by executing a program on more nodes at a slower gear rather than on fewer nodes at the fastest gear. We believe this will be important in the future, where a cluster may have heat limitations.

The reason why energy saving is possible is because of delays in the processor, where executing at a high frequency and voltage do not make the program execute faster, but does waste energy. This delay is due to (1) the processor waiting for the memory system to fetch a value or (2) the processor blocking awaiting a message from a remote processor.

Future Work. Armed with this preliminary information, there are several avenues for future study. First we will consider scaling down other components, such as the disk. Second, we will consider what we call the node bottleneck, in which a node reaches a synchronization point later than the rest of the nodes. A node bottleneck can occur for a variety of reasons, but the end result is that early-arriving nodes can be scaled down with little or no performance degradation. Third, we will develop a new MPI implementation that will automatically monitor executing programs and automatically reduce the energy gear appropriately. Finally, we need to experiment with large-scale programs; while we believe the NAS programs are representative, they are not industrial codes that number in the tens or hundreds of thousands of lines. Overall, the end goal of our research is the development of an energy- and performance-efficient parallel computing infrastructure. 


\section{Acknowledgments}

This research was supported in part by NSF award CCF 0234285. We give special thanks to Dan Smith for some of the low-level software and comments on early versions of the paper.

\section{References}

[1] N. Adiga et al. An overview of the BlueGene/L supercomputer. In Supercomputing 2002, Nov. 2002.

[2] M. Anand, E. Nightingale, and J. Flinn. Self-tuning wireless network power management. In Mobicom, Sept. 2003.

[3] P. Bohrer, E. Elnozahy, T. Keller, M. Kistler, C. Lefurgy, C. McDowell, and R. Rajamony. The case of power management in web servers. In R. Graybill and R. Melham, editors, Power Aware Computing. Kluwer/Plenum, 2002.

[4] E. V. Carrera, E. Pinheiro, and R. Bianchini. Conserving disk energy in network servers. In Proceedings of International Conference on Supercomputing, pages 86-97, San Fransisco, CA, 2003.

[5] J. S. Chase, D. C. Anderson, P. N. Thakar, A. Vahdat, and R. P. Doyle. Managing energy and server resources in hosting centres. In Symposium on Operating Systems Principles, pages 103-116, 2001.

[6] C. C. Corporation, I. Corporation, M. Corporation, P. T. Ltd. and T. Corporation. Advanced configuration and power interface specification, revision 2.0. July 2000.

[7] F. Douglis, P. Krishnan, and B. Bershad. Adaptive disk spin-down policies for mobile computers. In Proc. 2nd USENIX Symp. on Mobile and Location-Independent Computing, 1995.

[8] C. Ellis. The case for higher-level power management. In Proceedings of the 7th Workshop on Hot Topics in Operating Systems, March 1999.

[9] E. Elnozahy, M. Kistler, and R. Rajamony. Energy conservation policies for web servers. In USITS '03, 2003.

[10] E. M. Elnozahy, M. Kistler, and R. Rajamony. Energyefficient server clusters. In Workshop on Mobile Computing Systems and Applications, Feb 2002.

[11] K. Flautner, S. Reinhardt, and T. Mudge. Automatic performance-setting for dynamic voltage scaling. In Proceedings of the 7th Conference on Mobile Computing and Networking MOBICOM '01, July 2001.

[12] C. Gniady, Y. C. Hu, and Y.-H. Lu. Program counter based techniques for dynamic power management. In Proceedings of the 10th International Symposium on High-Performance Computer Architecture, Feb. 2004.

[13] D. Grunwald, P. Levis, K. Farkas, C. Morrey, and M. Neufeld. Policies for dynamic clock scheduling. In Proceedings of 4th Symposium on Operating System Design and Implementation, October 2000.

[14] S. Gurumurthi, A. Sivasubramaniam, M. Kandemir, and H. Franke. Dynamic speed control for power management in server class disks. In Proceedings of International Symposium on Computer Architecture, pages 169-179, June 2003.

[15] S. Gurumurthi, A. Sivasubramaniam, M. Kandemir, and H. Franke. Reducing disk power consumption in servers with DRPM. IEEE Computer, pages 41-48, Dec. 2003.

[16] T. Heath, E. Pinheiro, J. Hom, U. Kremer, and R. Bianchini. Application transformations for energy and performanceaware device management. In Proceedings of the 11th International Conference on Parallel Architectures and Compilation Techniques, Sept. 2002.

[17] D. P. Helmbold, D. D. E. Long, and B. Sherrod. A dynamic disk spin-down technique for mobile computing. In Mobile Computing and Networking, pages 130-142, 1996.
[18] C.-H. Hsu and U. Kremer. The design, implementation, and evaluation of a compiler algorithm for CPU energy reduction. In ACM SIGPLAN Conference on Programming Languages, Design, and Implementation, June 2003.

[19] R. Krashinsky and H. Balakrishnan. Minimizing energy for wireless web access with bounded slowdown. In Mobicom 2002, Atlanta, GA, September 2002.

[20] A. R. Lebeck, X. Fan, H. Zeng, and C. S. Ellis. Power aware page allocation. In Architectural Support for Programming Languages and Operating Systems, pages 105-116, 2000.

[21] C. Lefurgy, K. Rajamani, F. Rawson, W. Felter, M. Kistler, and T. W. Keller. Energy management for commerical servers. IEEE Computer, pages 39-48, Dec. 2003.

[22] R. J. Minerick, V. W. Freeh, and P. M. Kogge. Dynamic power management using feedback. In Workshop on Compilers and Operating Systems for Low Power, pages 6-1-610, Charlottesville, Va, Sept. 2002.

[23] O. Multisystems. http://www.orionmulti.com/.

[24] B. D. Noble, M. Satyanarayanan, D. Narayanan, J. E. Tilton, J. Flinn, and K. R. Walker. Application-aware adaptation for mobility. In Proceedings of the 16th ACM Symposium on Operating Systems and Principles, pages 276-287, October 1997.

[25] A. E. Papathanasiou and M. L. Scott. Energy efficiency through burstiness. In WMCSA, Oct. 2003.

[26] E. Pinheiro, R. Bianchini, E. V. Carrera, and T. Heath. Dynamic cluster reconfiguration for power and performance. In Compilers and Operating Systems for Low Power, Sept. 2001.

[27] E. Pinheiro, R. Bianchini, E. V. Carrera, and T. Heath. Load balancing and unbalancing for power and performance in cluster-based systems. In Workshop on Compilers and Operating Systems for Low Power, Sept. 2001.

[28] V. Sharma, A. Thomas, T. Abdelzaher, and K. Skadron. Power-aware QoS management in web servers. In 24th Annual IEEE Real-Time Systems Symposium, Cancun, Mexico, Dec. 2003.

[29] A. Vahdat, A. Lebeck, and C. Ellis. Every joule is precious: The case for revisiting operating system design for energy efficiency. SIGOPS European Workshop, 2000.

[30] J. S. Vetter. Performance analysis of distributed applications using automatic classification of communication inefficiencies. In International Conference on Supercomputing, pages 245-254, May 2000.

[31] J. S. Vetter and M. McCracken. Statistical scalability analysis of communication operations in distributed applications. In Principles and Practice of Parallel Programming, pages 123-132, June 2001.

[32] M. Warren, E. Weigle, and W. Feng. High-density computing: A 240-node beowulf in one cubic meter. In Supercomputing 2002, Nov. 2002.

[33] M. Weiser, B. Welch, A. J. Demers, and S. Shenker. Scheduling for reduced CPU energy. In Operating Systems Design and Implementation (OSDI '94), pages 13-23, 1994.

[34] F. C. Wong, R. P. Martin, R. H. Arpaci-Dusseau, and D. E. Culler. Architectural requirements and scalability of the NAS parallel benchmarks. In Proceedings of Supercomputing '99, Portland, OR, Nov. 1999.

[35] H. Zeng, C. S. Ellis, A. R. Lebeck, and A. Vahdat. Currentcy: Unifying policies for resource management. In USENIX 2003 Annual Technical Conference, June 2003.

[36] Q. Zhu, F. M. David, C. Devaraj, Z. Li, Y. Zhou, and P. Cao. Reducing energy consumption of disk storage using poweraware cache management. In Proceedings of the 10th International Symposium on High-Performance Computer Architecture (HPCA-10), Feb. 2004. 